Abstract 392 Table 1 PFS in higher-risk and lower-risk SOLO1 subgroups by investigator and BICR assessment

\begin{tabular}{|c|c|c|c|}
\hline & \multicolumn{2}{|c|}{ Median PFS, months } & \multirow{2}{*}{$\begin{array}{c}\text { HR }(95 \% \mathrm{Cl}) \\
\text { Olaparib vs Placebo }\end{array}$} \\
\hline & Olaparib & Placebo & \\
\hline \multicolumn{4}{|l|}{ Investigator } \\
\hline Higher risk & 39.0 & 11.1 & $0.34(0.24-0.48)$ \\
\hline Lower risk & NR & 21.9 & $0.33(0.20-0.52)$ \\
\hline \multicolumn{4}{|l|}{ BICR } \\
\hline Higher risk & NR & 11.3 & $0.32(0.22-0.49)$ \\
\hline Lower risk & NR & 19.2 & $0.29(0.17-0.50)$ \\
\hline
\end{tabular}

Disclosures Nicoletta Colombo: Consulting fees or advisory role from Roche/Genentech, PharmaMar, AstraZeneca, Clovis Oncology, Pfizer, MSD Oncology, Takeda, Tesaro, BioCad and GlaxoSmithKline; honoraria from Roche/Genentech, AstraZeneca, Tesaro and PharmaMar. William Bradley: Nothing to disclose. Kathleen Moore: Advisory board fees from Advaxis, Aravive, AstraZeneca, Clovis, Eisai, Genentech/ Roche, Immunogen, Janssen, Merck, Oncomed, Pfizer, Samumed, Tesaro, VBL Therapeutics. Antonio González-Martín: Speaker role/consulting fees from AstraZeneca, PharmaMar, Roche, Tesaro; consulting fees from Clovis Oncology, Genmab, Inmunogen, MSD, Pfizer. Giovanni Scambia: Nothing to disclose. Ana Oaknin: Consulting fees from AstraZeneca, Clovis Oncology, Genmab, Immunogen, PharmaMar, Roche, Tesaro. Michael Friedlander: Honoraria and speaker role/advisory boards from AstraZeneca; advisory board fees from Lilly, MSD, Takeda, Novartis; non-remunerated consulting for AbbVie; research funding from Beigene, AstraZeneca, Novartis. Elizabeth S. Lowe: Shareholder and employee of AstraZeneca. Philip Rowe: Shareholder and employee of AstraZeneca. Paul DiSilvestro: Consulting fees from AstraZeneca, Tesaro.

\section{REAL-LIFE RESPONSE TO STANDARD OF CARE IN ADVANCED OVARIAN CANCER - AN OBSERVATIONAL MULTI-NATIONAL COHORT STUDY (RESPONSE)}

${ }^{1}$ Christian Marth, ${ }^{2}$ Heini Lassus, ${ }^{3}$ Kristina Lindemann, ${ }^{4}$ Els Van Nieuwenhuyen, ${ }^{5}$ Anne Weng Ekmann-Gade, ${ }^{6}$ Jesper Hansen, ${ }^{7}$ Klaus Kaae Andersen, ${ }^{8}$ Jacob Korach. ${ }^{1}$ Medical University Innsbruck; Department of Obstetrics and Gynecology; ${ }^{2}$ Helsinki University Hospital and University of Helsinki; Department of Gynecology and Obstetrics; ${ }^{3}$ Oslo Universitetssykehus, Kreftklinikken; Avdeling for Gynekologisk Kreft; ${ }^{4}$ University Hospital Leuven; Department of Obstetrics and Gynecology; ${ }^{5}$ Juliane Marie Centre, Rigshospitalet; Department of Gynaecology; ${ }^{6}$ Astrazeneca; Medical Affairs; ${ }^{7}$ Astrazeneca; Medical Evidence; ${ }^{8}$ Sheba Medical Center

\subsection{6/ijgc-2020-ESGO.131}

Introduction/Background Recent randomized clinical trials have demonstrated convincing effects of integrating PARP inhibitors (PARPi) and combination of PARPi + bevacizumab (antiVEGF) into first line (1L) treatment of selected groups of advanced stage ovarian cancer (OC) patients. However, it remains unclear to which extent eligibility of PARPi treatment translates into a real-world setting, where the impact of patient heterogeneity and differences in national clinical practices may influence the potential for PARPi treatment. The aim of this study is to describe treatment strategies and outcomes of advanced OC; and to estimate the proportion of patients potentially eligible for 1L PARPi maintenance therapy and for concomitant anti-VEGF treatment practice using observational data in a multi-national setting (RESPONSE).

Methodology This international, multi-centre, observational study, includes real-world data on diagnostic work-up, standard of care, clinical outcomes and treatment for around 1000 patients with advanced OC $(\geq 120$ patients/country). Last index date is 1st April 2018, ensuring at least 20 months of follow-up. Potential PARPi eligibility is defined as having no macroscopic residual disease $(<1 \mathrm{~cm})$ following upfront surgery and/or having a clinical complete response/partial

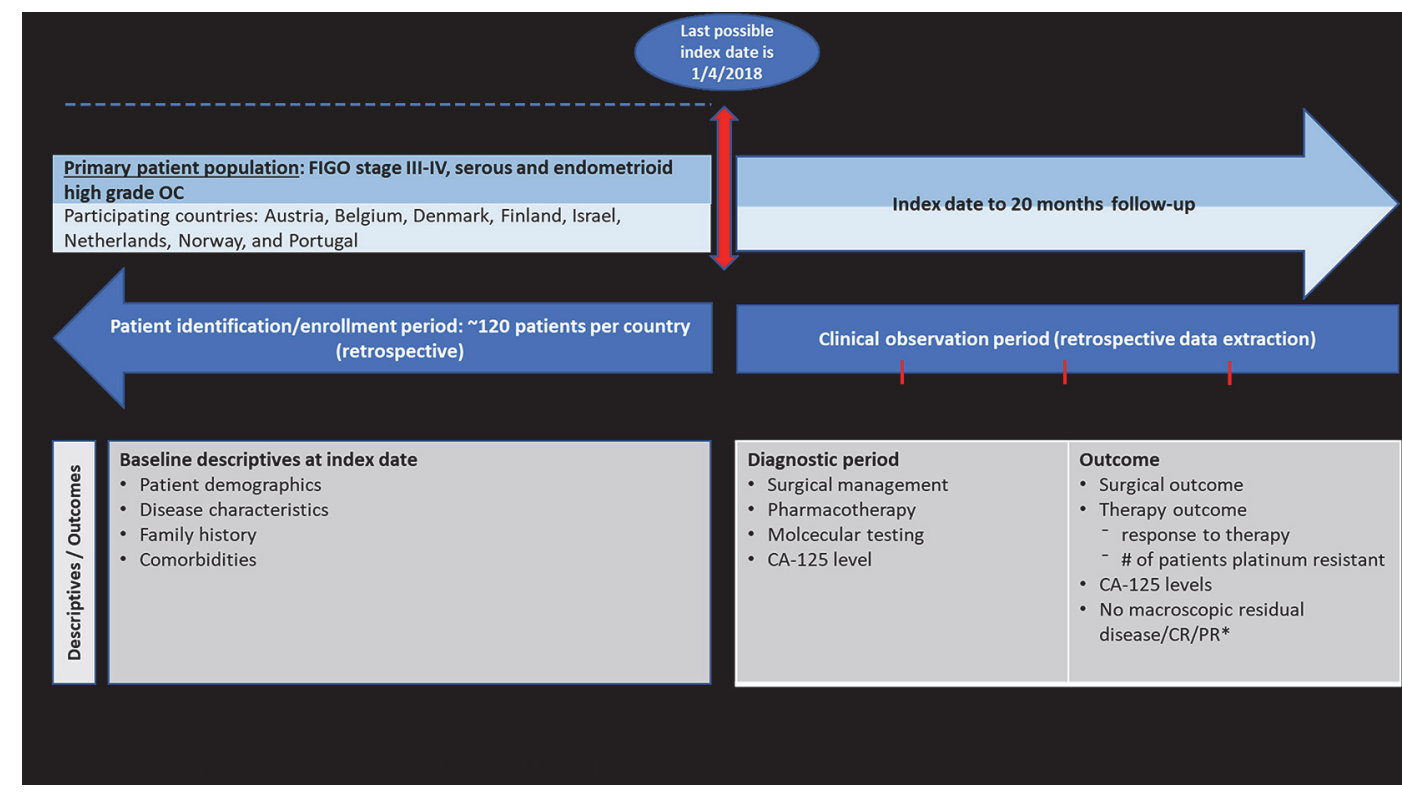

Abstract 405 Figure 1 
response $(\mathrm{CR} / \mathrm{PR})$ following completion of $1 \mathrm{~L}$ chemotherapy according to the label. Eligibility for PARPi treatment will be analysed prospectively by time-to-event by individual country, all countries combined, and in relation to treatment patterns. Finally, between-country variations will be described in relation to $1 \mathrm{~L}$ treatment patterns and national guidelines.

Results In total, eight countries, Austria, Belgium, Denmark, Finland, Israel, Netherlands, Norway, and Portugal, are included in the study (study flow chart; figure 1). Data collection is ongoing and will be finalized by Q1 2021. Inclusion of 120 patients per site will provide precise estimates of local PARPi eligibility (error margin of $4 \%$ ) and sufficient power to detect clinical meaningful differences in PARPi eligibility between any two countries.

Conclusion International real-world OC data is currently scarce. RESPONSE will add to the current knowledge regarding factors influencing eligibility to $1 \mathrm{~L}$ PARPi or PARPi + anti-VEGF maintenance treatment in individual countries, and enable mapping of patient characteristics and key variables in the $1 \mathrm{~L}$ treatment pathway, such as timing and outcome of surgery, including concomitant anti-VEGF treatment.

Disclosures Professor Christian Marth has received funded research from EU, FWF, AstraZeneca and Roche, Honoraria/ Expenses from Roche, Novartis, Amgen, MSD, Pharmamar, AstraZeneca, and Tesaro, and has performed Consulting/Advisory Boards for Roche, Novartis, Amgen, MSD, AstraZeneca, Pfizer, Pharmamar, Cerulean, Vertex, and Tesaro.

Dr Jacob Korach has nothing to disclose.

Dr Kristina Lindemann has acted as Consultant for AstraZeneca, Speaker for AstraZeneca and GSK, and participated in Advisory Boards for AstraZeneca and GSK.

Dr Anne Weng Ekmann-Gade has received research grants for the current trial

Dr E Van Nieuwenhuysen has nothing to disclose.

Dr Heini Lassus has nothing to disclose.

Dr Klaus Kaae Andersen is employed by AstraZeneca.

Jesper Hansen is employed by AstraZeneca.

\section{PROGNOSTIC VALUE OF THE TUMOR INFILTRATING LYMPHOCYTES AND THE NEUTROPHIL-TO-LYMPHOCYTE RATIO IN PATIENTS WITH ADVANCED OVARIAN CANCER}

${ }^{1}$ Beatriz Álvarez-Abril, ${ }^{1}$ Esmeralda García, ${ }^{1}$ Pilar de la Morena, ${ }^{1}$ Alejandra Ivars, ${ }^{1}$ Manuel Sánchez, ${ }^{2}$ Asunción Chaves, ${ }^{3}$ Francisco Pastor, ${ }^{1}$ Gema Marín, ${ }^{1}$ Francisco Ayala, ${ }^{1}$ Elena Garcia Martinez. 'Hospital General Universitario Morales Meseguer; Hematology and Medical Oncology; ${ }^{2}$ Hospital General Universitario Morales Meseguer; Pathology; ${ }^{3}$ Hospital General Universitario Reina Sofía; Pathology

\subsection{6/ijgc-2020-ESG0.132}

Introduction/Background Tumor infiltrating lymphocytes (TIL) and Neutrophil-to-lymphocyte ratio (NLR) have been objectified as independent prognostic factors in different tumours. There is not enough knowledge about the prognostic value of these two factors as a combination. This analysis aims to study the prognostic significance of TIL and NLR in patients with advanced ovarian cancer (OC).

Methodology Observational, single-center and retrospective analysis of a cohort of 135 patients with advanced stage OC treated between 2002 and 2019. Histological samples of ovarian tissue from the surgery of 92 patients were requested, with informed consent, and tissue microarrays (TMA) were constructed. For the TIL study, immunohistochemical staining of the TMA was made and a quantitative analysis was performed through the morphometric analysis of the lymphocytes. Samples were categorized in relation to total area as TIL $0=$ absence; $1=<25 \%$; $2=25-$ $50 \% ; 3=50-75 \% ; 4 \geq 75 \%$. Neutrophils and lymphocytes levels in peripheral blood at the diagnosis were collected to estimate NLR. Survival analysis was performed using Cox regression.

Results Average age 66 years (36-84 years). Median overall survival (OS): 56 months $(0.92-154 \mathrm{~m})$. FIGO stage: $80 \%$ III, 20\% IV. Histology: $87.2 \%$ papillary serous. ECOG: $18.5 \%$ ECOG 2 at diagnosis. Surgery: primary cytoreduction/after neoadjuvant treatment: 59/59 patients. TIL and NLR study: Both variables were not correlated (Spearman's rho: $-0.259, \mathrm{p}=$ 0.106). $75 \%$ of patients had TILCD3 infiltration $<25 \%$. Median $\mathrm{NLR}=3.72$. The univariate analysis showed a higher OS in patients with TILCD3> 25\% (HR 0.448, 95\% CI 0.19 - 1.02;
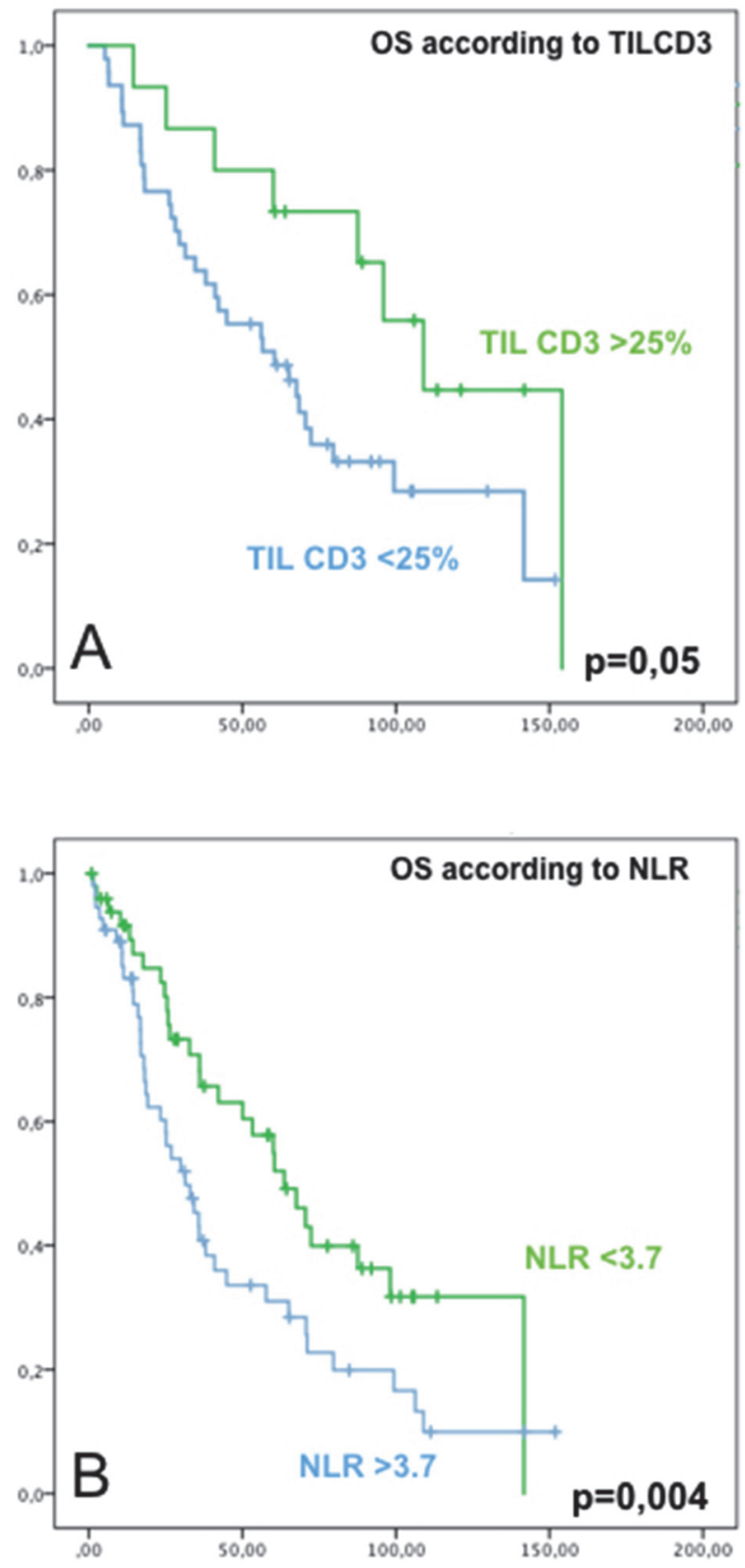

Abstract 413 Figure 1 